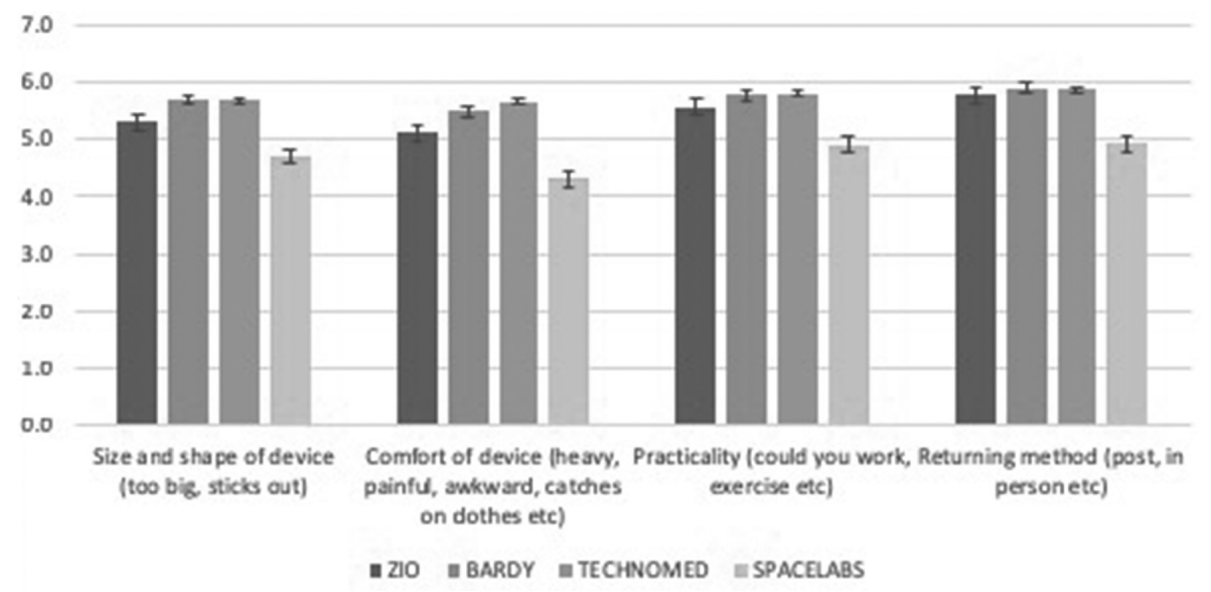

Abstract 43 Figure 1 Mean and Standard error values for Patient feedback, questions 3,4,7 and 8

\section{A SIGNIFICANT NUMBER OF PATIENTS WITH ATRIAL FIBRILLATION IN A LONDON MULTI-ETHNIC BOROUGH ARE NOT ANTICOAGULATED DESPITE A HIGH RISK SCORE}

${ }^{1}$ Abdul-Majeed Salmasi, ${ }^{2}$ Yasmin Bashir*. ${ }^{1}$ Central Middlesex Hospital; ${ }^{2}$ Imperial College London - Central Middlesex Hospital

10.1136/heartjnl-2019-BCS.42

A significant number of patients with Atrial Fibrillation in a London multi-ethnic borough are not anticoagulated despite a high-risk score

Background Atrial fibrillation (AF) is the commonest arrhythmia which, can have severe consequences if left untreated. Individuals with $\mathrm{AF}$ are at an increased risk of stroke. In order to minimise risks of any serious events occurring, it is important that patients are given the correct treatment. Oral anticoagulation can reduce the risk of stroke in these patients.

In an audit carried out in 2013, in 8 random GP practices across London borough of Brent, it was found that $51 \%$ of patients were not taking anticoagulation. This was a very significant, and extremely worrying finding and so there was a drive to improve patient education and advice to GPs. The audit was then repeated 2 years later and the percentage of patients not taking anticoagulation had dropped to $41 \%$.

After the introduction of the NICE guidelines in 2014, according to which, aspirin could no longer be prescribed on its own to reduce the risk of stroke, we decided to carry out another study.

Purpose To investigate the use of anticoagulation in AF patients in Brent, a multi-ethnic part of London and thus identify the proportion of patients who are at potential risk of developing stroke.

Methods A retrospective study across 8 randomly-selected general medical practices across the London borough of Brent was carried out to study the records of patients on the AF register. The following variables were recorded: age, gender, race, CHA2DS2VASc score and whether they were not taking anticoagulants and the reason why not taking anticoagulants.

Results There were 352 patients (188 males and 164 females) on the AF register. Only $66.2 \%$ of those patients were taking anticoagulants, with $47 \%$ of them taking a new oral anticoagulant. Of the 119 patients not taking anticoagulation, 73\% had a CHA2DS2VASc score of $>2$. In the 31 patients for whom anticoagulation was reported as 'not indicated', 29\% had a CHA2DS2VASc score of $>2$. Alongside this, in $13 \%$ of the patients who were not taking anticoagulation, no documentation was reported for the reason of not being anticoagulated. $33 \%$ of Caucasians, $10 \%$ of Afro-Caribbeans, $41 \%$ of Indo-Asians, and $44 \%$ of Arabs were not receiving anticoagulants.

Conclusion There is a significant proportion of patients who are on the AF register in general medical practice clinics with a CHA2DS2VASc score of $>2$ but are not taking anticoagulation. This puts many patients with $\mathrm{AF}$ at risk of ischaemic stroke. Physicians in general practice need to be made more aware of the high risk of patients with $\mathrm{AF}$ and the need for anticoagulation.

Conflict of Interest None

\section{COMPARING IMPACT OF AN E-LEARNING PACKAGE TO LECTURE-BASED TEACHING IN THE MANAGEMENT OF OF SUPRAVENTRICULAR TACHYCARDIA(SVT): A RANDOMIZED-CONTROLLED STUDY}

${ }^{1}$ Kevin Mohee* ${ }^{*}$ Hasan Haboubi, ${ }^{3}$ Majd Protty, ${ }^{4}$ Christopher Srinivasan, ${ }^{4}$ William Townend, ${ }^{2}$ Clive Weston. ${ }^{1}$ Department of Cardiology; ${ }^{2}$ Swansea University; ${ }^{3}$ Cardiff University; ${ }^{4}$ Emergency Department

\subsection{6/heartjnl-2019-BCS.43}

Introduction To compare the impact of e-learning package and theoretical teaching on the ability of both graduate and undergraduate medical students to learn the management of supraventricular tachycardia (SVT).

Methods We conducted a randomized controlled blinded study at two medical schools in Wales, UK. Participants included graduate-entry medical students from Swansea University and undergraduate medical students from Cardiff University. The intervention consisted of one hour of training using an e-learning package versus an hour of lecture based teaching. The outcome was comparison within each groups and between groups of mean scores using a pre-intervention and immediate post-intervention questionnaire. Another questionnaire was e-mailed after 2 weeks and mean scores were again compared to baseline, immediate post intervention between each groups and within each groups. The hypothesis 